\title{
The Relationship between Objectively Measured Physical Activity and Fundamental Motor Skills in 8 to 11 Years Old Children from the Czech Republic
}

\author{
Vlado Balaban ${ }^{1}$ \\ Affiliations: 'Palacky University, Faculty of Physical Culture, Olomouc, Czech Republic
}

Correspondence: V. Balaban, Palacky University, Faculty of Physical Culture, Trida Miru 117, 77900 Olomouc, Czech Republic. E-mail: vlado.balaban01@upol.cz

ABSTRACT The aim of this research was to explore the relationship between objectively measured physical activity and fundamental motor skills in 8-to-11-year-old children from the Czech Republic. The research sample consisted of 201 children (108 boys and 93 girls) aged 8-11 from Olomouc, Czech Republic. The Test of Gross Motor Development 2 was used to assess the level of children's fundamental motor skills. Furthermore, an ActiGraph GTX3 device accelerometer was used for the objective measurement of physical activity levels. The results have shown a low-to-medium correlation between moderate to vigorous physical activity and locomotor motor skills among the total sample, as well as between vigorous physical activity and object control skills in the sample of boys. Fundamental motor skills are essential factors for children's participation in organized and free-time physical activities. There is a commitment to develop fundamental motor skills in children, especially object control motor skills in girls.

KEY WORDS motor development, accelerometers, primary school children

\section{@MJSSMontenegro}

RELATIONSHIP BETWEEN PHYSICAL ACTIVITY AND SKILLS

http://mjssm.me/?sekcija=article\&artid=157

\section{Introduction}

Physical activity (PA) is defined as any bodily movement produced by skeletal muscles that results in energy expenditure (Caspersen, Powell, \& Christenson, 1985). Participation in regular PA could bring significant short- and long-term benefits for youth's health, especially in children's physical, social, cognitive, and psychological development (Janssen \& LeBlanc, 2010) e.g., improving children's health-related physical fitness (cardiorespiratory fitness and muscular strength) (Morrow, Tucker, Jackson, Martin, Greenleaf, \& Petrie, 2013); reducing body fat, improving bone health, reducing anxiety, and depression symptoms are related with higher levels of PA (Janssen \& LeBlanc, 2010). Despite these health benefits, many children and youngsters are not sufficiently physically active (Hallal et al., 2012). In contrast, physical inactivity (PI) is linked to an increased risk of several diseases: cardiovascular disease (CVD), hypertension, diabetes mellitus type 2, obesity, osteoporosis, depression, and colon cancer (U. S. Department of Health and Human Services [USDHHS], 1996). However, important factors for the determination of PA/PI among children are fundamental motor skills (FMS). In their conceptual model, Stodden et al. (2008) offered a system of relationships between motor competencies (MC), $\mathrm{PA}$, and the risk of obesity. This model proposes a reciprocal relationship between $\mathrm{MC}$ and PA and their influence on obesity. A positive spiral of engagement explains the lower risk of obesity within higher levels of PA and motor competence. Nevertheless, a negative spiral of disengagement causes a higher risk of obesity within lower levels of PA and motor competence. Children and youth who are better in terms of motor proficiency are more likely to participate in organized and free-time PA and build a healthy lifestyle later in their adult age (Stodden et al., 2008). Additionally, sedentary lifestyles appear more often among children with lower levels of motor proficiency; in adulthood, these children are more likely to avoid difficult movement patterns (Stodden \& Goodway, 2007).

FMS do not occur naturally with aging, and they must be learned as movement patterns (Hardy, ReintenReynolds, Espinel, Zask, \& Okely, 2012). FMS are the foundation for more complex types of PA and sports. 
FMS are mainly classified into two groups (Ulrich, 2000): 1) locomotor skills (i.e. run, jump, hop, leap, and slides); 2) object control skills (i.e. dribble, catch and kick the ball). Some studies that dealt with health benefits connected with FMS have discovered a strong evidence of positive dependence between FMS and PA among children (Lubans, Morgan, Cliff, Barnett, \& Okely, 2010). Some of these studies used a self-report method to display PA levels (Erwin \& Castelli, 2008). However, another systematic review study pointed out the absence of studies focused on the relationship between objectively measured PA and FMS through TGMD-2 among children in their middle childhood, especially in the age range from 8 to 11 years (Logan, Webster, Getchell, Pfeiffer, \& Robinson, 2015).

The aim of the research.

The aim of the present study is to discover the relationship between objectively measured PA and FMS among children in middle childhood from the Czech Republic. The present study also offers rare findings for this age group.

\section{Methods}

\section{Sample characteristics}

Data were collected from a convenient sample of 201 children: 108 boys and 93 girls (their average age $9.22 \pm 1.04$ years), without any health problems, physical or mental disability, attending three local primary schools in Olomouc, Czech Republic. The participation in the research was voluntary. From the participants and their parents/legal representatives, written permission prior to children's involvement in the research was obtained. Data collection was performed from December 2013 to March 2014. The study was approved by the Ethical Committee of the Faculty of Physical Culture, Palacký University, Olomouc (22/2014).

\section{Physical activity measurement}

An ActiGraph GT3X device accelerometer (Pensacola, FL, USA) was used to objectively measure children's daily PA. An accelerometer is very often taken as the "gold standard" for the objective tracking of field-based PA. It is a non-invasive method for children and eliminates the possibility of self-report biases (Bassett, Troiano, McClain, \& Wolff, 2015). The accelerometer provides detailed information about the time spent at different intensity levels, estimation of energy expenditure (EE) and the number of steps per unit of time (Cain, Conway, Adams, Husak, \& Sallis, 2013). The ActiGraph accelerometer generates output presented as activity counts per unit of time. For this study, the accelerometer data were recorded in 60s intervals. Based on the count of thresholds, it is possible to estimate the total time spent in PA of various intensity. For this study, the cut-off points (CoP) proposed by Freedson, Pober and Janz (2005) were applied. According to these authors, the intensity of PA is divided into: sedentary PA (SPA): 0-149 counts/min (cpm); light PA (LPA): 150-499 cpm; moderate (MPA): 500-3999 cpm; vigorous PA (VPA): 4000-7599 cpm; and very vigorous PA (VVPA): $\geq 7600 \mathrm{cpm}$. Tracking of children's PA with ActiGraph accelerometers has a proper validity and reliability (de Vries, Bakker, Hopman-Rock, Hirasing, \& van Mechelen, 2006). For the present study, the ActiGraph GT3X was placed in a pouch which was attached to a belt that children wore around their waists. Children were asked to wear the devices from their waking up and throughout the day and to take it off only when swimming, bathing, and sleeping. Children wore the devices for three weekdays and one weekend day (Puyau, Adolph, Vohra, \& Butte, 2002). The period of 10 or more hours of wearing the ActiGraph GT3X device was used for further data analysis (Troiano, Berrigani, Dodd, Masse, Tilert, \& McDowell, 2008).

\section{FMS assessment}

For the assessment of children's FMS, the Test of Gross Motor Development-2 (TGMD-2) was used. The TGMD-2 was designed to assess 12 FMS, and it is further divided into two subtests: the test of locomotor skills (run, gallop, hop, leap, jump, and slide) and test of object control skills (two-hand strike, stationary dribble, catch, kick, throw, and underhand roll). Every child performs each skill twice, and their performance is evaluated based on the performance criteria. An observer evaluates the performance of every skill according to four to six skill criteria. Skills are scored with units 0 or 1 , which indicates the absence or presence of the tested criteria. The highest total raw score for each subtest (locomotor and object control skills) is 48 points. Raw scores for two subtests are added and converted to percentiles and to gross motor quotient (GMQ) for each child. Reliability and validity of the TGMD-2 among young children have been documented (Ulrich, 2000). Data used for analysis included the locomotor (LM) subtest raw score, the object control (OC) skills, LM and OC skills overall (i.e., LM subtest raw score + OC subtest raw score) and GMQ.

\section{Statistical analysis}

All statistical analyses were processed in STATISTICA 12 (StatSoft, Prague, Czech Republic). The results of each sub-test were presented by means and standard deviations. The relationship between volume and intensity of PA and FMS were quantified using Pearson's correlation coefficient $r$. The relationship between the monitored variables was interpreted by Cohen (1992): $r=0$ - complete independence; $0.00<r<0.20$ - very weak dependence; $0.20 \leq \mathrm{r}<0.40$ - low dependence; $0.40 \leq \mathrm{r}<0.70$ - medium dependence; $0.70 \leq \mathrm{r}<0.90$ - high dependence; $0.90 \leq r<1.00$ - very high dependence; $r=1-$ total dependence. Significance was set at $p<0.05$ for all tests. 
Results

The average GMQ score for total sample regardless of gender shows that approximately half of children (52\%) have average developed FMS. Above average developed FMS occurred in $32 \%$ of the entire sample. A low level of motor development is present in $5 \%$ of the sample, and $3 \%$ of the sample has superior motor development. Boys performed better on the OC skills and LM and OC skills overall. In contrast, girls performed better in LM skills and GMQ (Table 1).

\begin{tabular}{|c|c|c|c|c|c|c|}
\hline & \multicolumn{2}{|c|}{$\begin{array}{c}\text { Boys ( } n=108) \\
8.00-11.99 \text { yrs. }\end{array}$} & \multicolumn{2}{|c|}{$\begin{array}{c}\text { Girls ( } n=93) \\
8.00-11.99 \text { yrs. }\end{array}$} & \multicolumn{2}{|c|}{$\begin{array}{c}\text { All }(N=201) \\
8.00-11.99 \text { yrs. }\end{array}$} \\
\hline & $M$ & $S D$ & $M$ & $S D$ & $M$ & $S D$ \\
\hline LM skills [points] & 46.29 & 3.19 & 46.68 & 1.69 & 46.47 & 2.90 \\
\hline OC skills [points] & 42.64 & 5.38 & 39.58 & 5.38 & 41.22 & 5.47 \\
\hline LM and OC skills overall [points] & 89.06 & 6.90 & 86.09 & 6.47 & 87.68 & 6.85 \\
\hline GMQ [points] & 104.28 & 12.03 & 104.96 & 11.71 & 104.59 & 11.90 \\
\hline SPA [min/day] & 1038.68 & 96.21 & 1019.23 & 185.02 & 1029.68 & 144.20 \\
\hline LPA [min/day] & 138.78 & 27.31 & 138.14 & 24.48 & 138.48 & 25.79 \\
\hline MPA [min/day] & 206.81 & 54.01 & 197.82 & 44.25 & 202.66 & 49.82 \\
\hline MVPA [min/day] & 220.32 & 57.31 & 210.65 & 49.90 & 215.85 & 54.09 \\
\hline VPA [min/day] & 11.77 & 9.54 & 9.69 & 7.45 & 10.81 & 8.67 \\
\hline VVPA [min/day] & 1.24 & 4.53 & 1.46 & 3.03 & 1.34 & 3.90 \\
\hline Steps [steps/day] & 10456 & 2931.14 & 9664 & 2592.57 & 10090.21 & 2800.90 \\
\hline
\end{tabular}

Note. $\mathrm{n}=$ the sample size; $\mathrm{M}=$ mean; $\mathrm{SD}=$ standard deviation, LM skills = Locomotor skills, OC skills = Object Control skills, $\mathrm{LM}$ and OC skills overall = Locomotor and Object control skills overall, GMQ = Gross Motor Quotient, SPA = sedentary PA, LPA = light intensity of PA, MPA = moderate intensity of PA, MVPA = moderate to vigorous intensity of PA, VPA = vigorous intensity of PA, VVPA = very vigorous intensity of PA.

According to ActiGraph GT3X data, the children's sample, regardless of gender, spent $60 \%$ of the monitored time in SPA. In the zone of MVPA children spent $13.25 \%$ of the monitored time. A similar amount of time was spent in LPA (12.50\%) and MPA (12.44\%). Children spent the shortest time in VPA (0.66\%) and VVPA (0.08\%) (Table 1). A Pearson product-moment correlation coefficient was computed to assess the relationship between PA levels and indicators of FMS. In the total sample, regardless of gender, very weak positive correlation between the following variables was found: VPA correlated with LM skills ( $r=0.17 ; p<0.05)$, with LM and OC skills overall $(r=0.17 ; \mathrm{p}<0.05)$, with OC skills $(\mathrm{r}=0.16 ; \mathrm{p}<0.05)$ and with GMQ $(\mathrm{r}=0.16 ; \mathrm{p}<0.05)$. Number of steps/day correlated with OC skills $(\mathrm{r}=0.19 ; \mathrm{p}<0.01)$, with LM and OC skills overall $(\mathrm{r}=0.17 ; \mathrm{p}<0.05)$ and finally with GMQ $(r=0.15 ; \mathrm{p}<0.05)$. In the boys' sample, statistically significant positive correlation was found between VPA and all indicators of FMS: LM skills $(r=0.19 ; \mathrm{p}<0.05)$, OC skills $(\mathrm{r}=0.24 ; \mathrm{p}<0.05)$, LM and OC skills overall $(\mathrm{r}=0.28 ; \mathrm{p}<0.01)$ and GMQ $(\mathrm{r}=0.30 ; \mathrm{p}<0.01)$. Similar weak correlation was four between the number of steps/day and OC skills $(r=0.22 ; \mathrm{p}<0.05)$, LM and OC skills overall $(\mathrm{r}=0.21 ; \mathrm{p}<0.05)$, and GMQ $(\mathrm{r}=0.23 ; \mathrm{p}<0.05)$. There were no significant correlations in the sample of girls.

\section{Discussion}

There is a problem of decreasing PA among children and adolescents in the Czech Republic. The present study indicates that boys $(\hat{})$ ) were more physically active than girls $(+$ ) in all levels of PA intensity except the VVPA. If we consider the recommendations from Tudor-Locke et al. (2011), 80\% of the boys from our sample met these recommendations (13,000 steps/day for $\widehat{\delta})$. Regarding the girls' sample, recommendations are found in an even larger percentage (88\%: 11,000 steps/day for + ). According to data from 2010, children aged 6-11 years, achieved average 10,128 steps/day $(+)$ and 10,600 steps/day ( $\left({ }^{\top}\right.$ ) (Sigmundová \& Sigmund, 2015), which is an average of 600 steps/day (for + ) and 144 steps/day (for $\hat{\jmath}$ ) more than in the present study. This finding corresponds with the previous study conducted by Cameron, Craig, Bauman, and Tudor-Locke (2016). These authors in their longitudinal study of Canadian children (regardless of gender) aged from 6 to 11 years of age have found a significantly negative trend in the number of steps/day (10,997 steps/day from 2005 to 2006 versus 10,331 steps/day from 2012 to 2014). Because 12,000 steps/day correspond to approximately $60 \mathrm{~min} /$ day MVPA (Colley, Janssen, \& Tremblay, 2012), it may be stated that $84 \%$ of the total sample met a recommendation for at least $60 \mathrm{~min} /$ day of MVPA. The recommended $90 \mathrm{~min} /$ day of MVPA (Sigmund, El Ansari, \& Sigmundová, 2012) based on the number of steps/day, were performed by approximately $56 \%$ of children from the present study. Based on the results of the cpm of the accelerometer and their distribution by the CoP from Freedson et al. (2005), we can say that $100 \%$ of our sample met the recommended 90 min/day of MVPA (Sigmund et al., 2012). With a sample of 288 children from the US (aged 6 to 11 years), in their study, Troiano et al. (2008) showed that $42 \%$ of children (48.90\% of boys and $34.70 \%$ of girls) met a recommendation for $60 \mathrm{~min} /$ day MVPA. The average time spent in the MVPA zone was $84.90 \mathrm{~min} /$ day $\left(95.40 \mathrm{~min} /\right.$ day for $\sigma^{\lambda}$ and 75.20 for +). A sample from another study, consisting of 169 children from Olomouc $(\mathrm{M}=9.92$ years), spent an average of $43.28 \mathrm{~min} /$ day of MVPA (Šnoblová, Jakubec, Sigmund, \& Sigmundová, 2015). 
We assume that the reason for the large difference between the time spent in the MVPA is caused by different devices used for the objective monitoring of $\mathrm{PA}$ and, consequently, by selecting the $\mathrm{CoP}$ for the PA intensity zones. These findings are in accordance with Vanderloo, Di Cristofaro, Proudfoot, Tucker, and Timmons (2016) who underscore the difficulties in evaluating the results of the objective monitoring of PA measured for the same children's sample with different devices. Additionally, some studies have proven that the selection of CoP could notably change an average of MVPA and the percentage of children who met PA recommendations (Beets, Bornstein, Dowda, \& Pate, 2011). An average GMQ score of the total sample has shown that approximately half of children (52\%) have average-developed FMS. Above average FMS occurred in $32 \%$ of the total sample. Low levels of motor development are present in $5 \%$ of the sample while superior motor development occurred in $3 \%$ of the sample.

The results of the present study indicate that boys achieved higher results in OC skills and the LM and OC skills overall. In contrast, girls achieved higher results from the LM skills and GMQ. These results confirm the trends from other studies, in which, with regard to gender, boys have shown significantly higher scores in OC skills and LM and OC skills overall (Burns, Brusseau, \& Hannon, 2017). Girls have shown better scores in LM skills (Burns et al., 2017; Slykerman, Ridgers, Stevenson, \& Barnett, 2016). The OC skills tested in the present study are typical skills included in many ball games, which are more popular among boys than among girls. However, some LM skills are mostly taught during dance classes, which are more popular among girls. The relationships between PA and OC skills were more powerful than the relationships between PA and LM skills. Similar results were presented by Hume et al. (2008). Relations between the levels of PA and levels of FMS in the total sample regardless of gender are partially in alignment with the results of the research conducted by Lopes, Maia, Rodrigues, and Malina (2012), especially in the relationship between VPA and LM and OC skills overall $(\mathrm{r}=0.18)$ among children aged from 8 to 10 years. The same authors presented a weak dependence $(r=0.22)$ between the total level of FMS and MVPA $(r=0.30)$. In the present study, no significant correlations were found between these two variables. In contrast to the boys' sample from the present study, Hume et al. (2008) reported a weak correlation between the total level of FMS and MPA $(r=0.21)$, VPA $(r=0.25)$ and MVPA ( $r=0.24)$. In comparison with those results, the boys' sample from the present study demonstrated a significantly weak correlation between the VPA and LM and OC skills overall and between VPA and OC skills. Hume et al. (2008) also presented low dependence in the girls' sample between VPA and LM skills $(r=0.29)$ and between VPA and LM and OC skills overall $(r=0.21)$. However, in the girls' sample from the present study, there were no significant correlations between any component of FMS and any level of PA intensity. The absence of significantly positive correlation between the levels of PA and the levels of FMS in the present study is unexpected, considering previous findings indicating that these skills are related to PA in girls (Burns et al., 2017; Castelli \& Valley, 2007).

Findings from the present study did not support a strong relationship as other studies did. We believe that the difference was shown, because in the research conducted by Hume et al. (2008) only five items for the evaluation of the level of FMS (three subtests for OC skills and two subtests for LM skills) were used, which could be explained with better results in the overall OC skills score in the sample from Hume et al. (2008) especially in the girls' sample. However, we agree with the statement of Wrotniak, Epstein, Dorn, Jones, and Kondilis (2006) that the children with better developed FMS choose to participate in a wider range of PA, which gives them the opportunity for plenty of movement experience. Over time, that leads to increasing amounts and intensities of PA.

\section{Conclusion}

With regard to gender, the study sample completely met the recommendations for PA set for primary school children (90 min/day MVPA) based on the cpm recorded by the ActiGraph GT3X device. In contrast, $80 \%$ of boys and $88 \%$ of girls met the recommendation based on the number of steps/day (13,000 steps/day for boys and 11,000 steps/day for girls). This difference could be explained by the selected CoP for individual PA intensity zones, where specific CoP can significantly distort the values on time spent in each of the PA intensity zones. The low level of PA can be explained by the results of the evaluation of the level of FMS. Based on the level of FMS assessed by TGMD-2, 52\% of the total sample is at an average level of gross motor development ( $44 \%$ of the boys' and $58 \%$ of the girls' sample, respectively). In conclusion, it could be underlined that the promotion of FMS in both genders is a significant factor for increasing the levels of children's PA.

\section{References}

Bassett, D. R., Troiano, R. P., McClain, J. J., Wolff, D. L. (2015). Accelerometer-based physical activity: Total volume per day and standardized measures. Medicine and Science in Sports and Exercise, 47(4), 833-838. doi: $10.1249 / \mathrm{mss} .0000000000000468$

Beets, M. W., Bornstein, D., Dowda, M., \& Pate, R. R. (2011). Compliance with national guidelines for physical activity in US preschoolers: Measurement and interpretation. Pediatrics, 127(4), 658-664. doi: 10.1542/ peds.2010-2021d

Burns, R. D., Brusseau, T. A., \& Hannon, J. C. (2017). Multivariate associations among health-related fitness, physical activity, and TGMD-3 test items in disadvantaged children from low-income families. Perceptual and Motor Skills, 124(1), 86-104. doi: 10.1177/0031512516672118 
Cain, K. L., Conway, T. L., Adams, M. A., Husak, L. E., \& Sallis, J. F. (2013). Comparison of older and newer generations of ActiGraph accelerometers with the normal filter and the low frequency extension. International Journal of Behavioral Nutrition and Physical Activity, 10(1), 51-57. doi: 10.1186/1479-5868-10-51

Cameron, C., Craig, C. L., Bauman, A., \& Tudor-Locke, C. (2016). CANPLAY study: Secular trends in steps/ day amongst 5-19-year-old Canadians between 2005 and 2014. Preventive Medicine, 86(1), 28-33. doi: 10.1016/j.ypmed.2015.12.020

Caspersen, C. J., Powell, K. E., \& Christenson, G. M. (1985). Physical activity, exercise and physical fitness: definitions and distinctions for health-related research. Public Health Reports, 100(2), 126-131.

Castelli, D. M., \& Valley, J. A. (2007). Chapter 3: The relationship of physical fitness and motor competence to physical activity. Journal of Teaching in Physical Education, 26(4), 358- 374. doi: 10.1123/jtpe.26.4.358

Cohen, J. (1992). A power primer. Psychological Bulletin, 112(1), 155-159. doi: 10.1002/0471722227.ch14

Colley, R. C., Janssen, I., \& Tremblay, M. S. (2012). Daily step target to measure adherence to physical activity guidelines in children. Medicine and Science in Sports Exercise, 44(5), 977-982. doi: 10.1249/ mss.0b013e31823f23b1

de Vries, S. I., Bakker, I., Hopman-Rock, M., Hirasing, R. A., \& van Mechelen, W. (2006). Clinimetric review of motion sensors in children and adolescents. Journal of clinical epidemiology, 59(7), 670-680. doi: 10.1016/j.jclinepi.2005.11.020

Erwin, H. E., \& Castelli, D. M. (2008). National physical education standards: A summary of student performance and its correlates. Research Quarterly for Exercise and Sport, 79(4), 495-505. doi: 10.5641/1 93250308x13086832906670

Freedson, P. S., Pober, D., \& Janz, K. F. (2005). Calibration of accelerometer output for children. Medical Science of Sports Exercise, 37(Suppl. 11), 523-530. doi: 10.1249/01.mss.0000185658.28284.ba

Hallal, P. C., Andersen, L. B., Bull, F. C., Guthold, R., Haskell, W., Ekelund, U., \& Lancet Physical Activity Series Working Group. (2012). Global physical activity levels: surveillance progress, pitfalls, and prospects. The Lancet, 380(9838), 247-257. doi: 10.1016/s0140-6736(12)60646-1

Hardy, L. L., Reinten-Reynolds, T., Espinel, P., Zask, A., \& Okely, A. D. (2012). Prevalence and correlates of low fundamental movement skill competency in children. Pediatrics, 130(2), 390-398. doi: 10.1542/ peds.2012-0345d

Hume, C., Okely, A., Bagley, S., Telford, A., Booth, M., Crawford, D., \& Salmon, J. (2008). Does weight status influence associations between children's fundamental movement skills and physical activity? Research Quarterly for Exercise and Sport, 79(2), 158-165. doi: 10.5641/193250308x13086753543374

Janssen, I., \& LeBlanc, A. G. (2010). Systematic review of the health benefits of physical activity and fitness in school-aged children and youth. International Journal of Behavioral Nutrition and Physical Activity, 7(40), 1-16. doi: 10.1186/1479-5868-7-40

Logan, S. W., Webster, E. K., Getchell, N., Pfeiffer, K. A., \& Robinson, L. E. (2015). Relationship between fundamental motor skill competence and physical activity during childhood and adolescence: A systematic review. Kinesiology Review, 4(4), 416-426. doi: 10.1123/kr.2013-0012

Lopes, V. P., Maia, J. A. R., Rodrigues, L. P., \& Malina, R. M. (2012). Motor coordination, physical activity and fitness as predictors of longitudinal change in adiposity during childhood. European Journal of Sport Science, 12(4), 384-391. doi: 10.1080/17461391.2011.566368

Lubans, D. R., Morgan, P. J., Cliff, D. P., Barnett, L. M., \& Okely, A. D. (2010). Fundamental movement skills in children and adolescents. Sports Medicine, 40(12), 1019-1035. doi: 10.2165/11536850-000000000-00000

Morrow, J. R., Tucker, J. S., Jackson, A. W., Martin, S. B., Greenleaf, C. A., \& Petrie, T. A. (2013). Meeting physical activity guidelines and health-related fitness in youth. American Journal of Preventive Medicine, 44(5), 439-444. doi: 10.1016/j.amepre.2013.01.008

Puyau, M. R., Adolph, A. L., Vohra, F. A., \& Butte, N. F. (2002). Validation and calibration of physical activity monitors in children. Obesity Research, 10(3), 150-157. doi: 10.1038/oby.2002.24

Sigmundová, D., \& Sigmund, E. (2015). Trendy v pohybovém chování českých dětí a adolescentů. Olomouc: Univerzita Palackého.

Sigmund, E., El Ansari, W., \& Sigmundová, D. (2012). Does school-based physical activity decrease overweight and obesity in children aged 6-9 years? A two-year non-randomized longitudinal intervention study in the Czech Republic. BMC Public Health, 12(570), 1-13. doi: 10.1186/1471-2458-12-570

Slykerman, S., Ridgers, N. D., Stevenson, C., \& Barnett, L. M. (2016). How important is young children's actual and perceived movement skill competence to their physical activity? Journal of Science and Medicine in Sport, 19(6), 488-492. doi: 10.1016/j.jsams.2015.07.002

Stodden, D., \& Goodway, J. D. (2007). The dynamic association between motor skill development and physical activity. Journal of Physical Education, Recreation, and Dance, 78(8), 33-49. doi: 10.1080/07303084.2007.10598077

Stodden, D. F., Goodway, J. D., Langendorfer, S. J., Roberton, M. A., Rudisill, M. E., Garcia, C., \& Garcia, L. E. (2008). A developmental perspective on the role of motor skill competence in physical activity: An emergent relationship. Quest, 60(2), 290-306. doi: 10.1080/00336297.2008.10483582

Šnoblová, R., Jakubec, L., Sigmund, E., \& Sigmundová, D. (2015). Srovnání školní a celodenní pohybové aktivity 9-10letých děvčat a chlapců. Tělesná kultura, 38(1), 92-106. doi: 10.5507/tk.2015.005

Troiano, R. P., Berrigani, D., Dodd, K. W., Mâsse, L. C., Tilert, T., \& McDowell, M. (2008). Physical activity in 
the United States measured by accelerometer. Medicine and Science in Sports and Exercise, 40(1), 181-188. doi: $10.1249 / \mathrm{mss} .0 \mathrm{~b} 013 \mathrm{e} 31815 \mathrm{a} 51 \mathrm{b3}$

Tudor-Locke, C., Craig, C. L., Beets, M. W., Belton, S., Cardon, G. M., Duncan, S., ... Blair, S. N. (2011). How many steps/day are enough? For children and adolescents. International Journal of Behavioral Nutrition and Physical Activity, 8(78), 1-14. doi: 10.1186/1479-5868-8-78

Ulrich, D. A. (2000). The test of gross motor development. (2nd ed.) Austin, TX: PRO-ED.

United States Department of Health and Human Services (1996). Physical activity and health: A report of the surgeon general. Department of Health and Human Services. Retrieved from https://www.cdc.gov/ nccdphp/sgr/pdf/execsumm.pdf

Vanderloo, L. M., Di Cristofaro, N. A., Proudfoot, N. A., Tucker, P., Timmons, \& Timmons, B. W. (2015). Comparing the Actical and Actigraph approach to measuring young children's physical activity levels and sedentary time. Pediatric Exercise Science, 28(1), 133-142. doi: 10.1123/pes.2014-0218

Wrotniak, B. H., Epstein, L. H., Dorn, J. M., Jones, K. E., \& Kondilis, V. A. (2006). The relationship between motor proficiency and physical activity in children. Pediatrics, 118(6), 1758-1765. doi: 10.1542/peds.20060742 